\title{
Avaliação da aplicabilidade de sistema fotovoltaico conectado à rede em uma granja no Mato Grosso
}

O desenvolvimento econômico e a promoção da qualidade de vida da população vêm despertando o aumento da preocupação quanto ao suprimento da demanda energética mundial. 0 esgotamento de fontes não renováveis de energia como o carvão mineral, petróleo e gás natural, além do cenário atual frente às mudanças climáticas, com uma grande parcela de emissões de gases de efeito estufa pelo setor energético, tende a incentivar novos investimentos em fontes renováveis de energia, dentre elas, a energia solar. Este estudo visa dimensionar e avaliar a capacidade de geração de energia elétrica a partir de um Sistema Fotovoltaico Conectado à Rede - SFVCR, para desenvolvimento das atividades de uma granja produtora de aves no estado do Mato Grosso, frente aos impactos ambientais intrínsecos ao sistema. De acordo com informações acerca do consumo de energia do empreendimento, bem como segundo dados de do Mato Grosso, frente aos impactos ambientais intrínsecos ao sistema. De acordo com informações acerca do consumo de energia do empreendimento, bem como segundo dados de
irradiação solar incidente no plano, disponibilizados pelo banco de dados do Atlas Brasileiro de Energia Solar, foi possível projetar um sistema fotovoltaico para realizar o abastecimento de energia elétrica do empreendimento. Baseado na potência do sistema dimensionado e capacidade de geração de energia elétrica, foi calculada a estimativa de emissões de gases de efeito estufa evitadas durante a operação do sistema, segundo dados publicados pelo Ministério da Ciência, Tecnologia, Inovação e Comunicações (MCTIC). Os resultados indicam a viabilidade para implantação do SFVCR no local, tendo em vista a disponibilidade de área para implantação, incidência de irradiação solar constante, permitindo a geração de energia elétrica ao longo do ano e compensação junto à concessionária local, de acordo com a Resolução Normativa no 482/2012 da ANEEL. Ainda, foi demonstrado que, caso o sistema seja efetivamente implantado, a emissão de gases de efeito estufa a partir do consumo de energia pela granja, seria minimizada em pelo menos 17 tCO2 equivalente.

Palavras-chave: Energia Solar; Sistema Fotovoltaico; Mudanças Climáticas.

\section{Evaluation of the applicability of a grid-connected photovoltaic system in a farm in the state of Mato Grosso}

\begin{abstract}
Economic development and the promotion of the quality of life of the population has been raising the concern about the supply of world energy demand. The depletion of non-renewable sources of energy such as coal, oil and natural gas, as well as the current scenario of climate change, with a large share of greenhouse gas emissions by the energy sector, tends to encourage new investments in renewable sources of energy, among them, solar energy. This study aims to evaluate the electric power generation capacity of a Grid-connected Photovoltaic System, to develop the activities of a poultry farm in the state of Mato Grosso, in view of the environmental impacts intrinsic to the system. According to information about the energy consumption of the place, as well as data from solar radiation incident in the surface, available on the database of the Brazilian Atlas of Solar Energy, it was possible to design a photovoltaic system to supply the place, as well as data from solar radiation incident in the surface, available on the database of the Brazilian Atlas of Solar Energy, it was possible to design a photovoltaic system to supply
the farm's electric energy. Based on the power of the scaled system and the capacity to generate electric energy the estimation of greenhouse gas emissions avoided during the operation of the system was calculated, according to data published by the Ministry of Science, Technology, Innovation and Communications. The results indicate the viability for the implantation of the Grid-connected Photovoltaic System in the place, considering the availability of area for implantation, constant solar irradiation incidence, allowing the generation of electric energy throughout the year and compensation to the local concessionaire, according to ANEEL's Normative Resolution № 482/2012. In addition, it was demonstrated that, if the system were effectively implemented, the emission of greenhouse gases from energy consumption by the farm would be minimized in at least 17 tCO2 equivalent.
\end{abstract}

Keywords: Solar Energy; Photovoltaic System; Climate Change.

Topic: Desenvolvimento, Sustentabilidade e Meio Ambiente

Reviewed anonymously in the process of blind peer.

Mayara Luiza Paiva (i)

Universidade Tecnológica Federal do Paraná, Brasil

http://lattes.cnpq.br/3512561352952269

http://orcid.org/0000-0002-6081-5469

mayalpaiva@gmail.com

Julio César Rodrigues de Azevedo (iD

Universidade Tecnológica Federal do Paraná, Brasil

http://lattes.cnpq.br/8987771365126082

http://orcid.org/0000-0001-5358-0713

jcrazevedo@utfpr.edu.br

\section{Jair Urbanetz Júnior (DD}

Universidade Tecnológica Federal do Paraná, Brasil

http://lattes.cnpq.br/2654512569280452

http://orcid.org/0000-0001-9355-1730

urbanetz@utfpr.com.br

d

DOI: 10.6008/CBPC2179-6858.2020.001.0025
Received: $\mathbf{1 4 / 1 2 / 2 0 1 9}$

Approved: 17/01/2020
Referencing this:

PAIVA, M. L.; AZEVEDO, J. C. R.; URBANETZ JÚNIOR, J.. Avaliação da aplicabilidade de sistema fotovoltaico conectado à rede em uma granja no Mato Grosso. Revista Ibero Americana de Ciências Ambientais, v.11, n.1, p.274-288, 2020. DOI:

http://doi.org/10.6008/CBPC2179-6858.2020.001.0025 


\section{INTRODUÇÃO}

O crescimento populacional e a melhoria dos padrões da vida de países em desenvolvimento, vem sendo acompanhados da preocupação em suprir a demanda energética, tendo em vista a perspectiva de esgotamento das fontes não-renováveis de energia como o petróleo. 0 contexto energético é um dos assuntos de maior importância atualmente, considerando a relação bem definida entre qualidade de vida da população e consumo de energia (MARTINS et al., 2008).

Mesmo considerando a produção de energia já obtida através de fontes renováveis, como hidrelétrica e biomassa, a necessidade de introduzir recursos complementares na matriz energética mundial ainda perdura, considerando que podem haver longos períodos de estiagem que prejudiquem o sistema (MARTINS et al., 2008). Tendo em vista que o sol é a fonte primária mais abundante no planeta, o aproveitamento da irradiação solar para produção de energia elétrica através do efeito fotovoltaico, está tomando espaço nos estudos atuais (PINHO et al., 2014).

O uso de sistemas fotovoltaicos, principalmente quando conectados à rede elétrica pública, estão em ascensão no mundo todo pela sua geração de energia renovável, de forma distribuída e próxima ao ponto de consumo (TIEPOLO, 2015). Este sistema possui uma elevada produtividade, onde toda energia gerada é utilizada, sendo pela própria instalação onde o sistema está implantado, ou pela injeção na rede pública (URBANETZ JR., 2010).

Este estudo visa avaliar a aplicabilidade de um Sistema Fotovoltaico Conectado à Rede (SFVCR), tendo em vista a eficiência para suprir o consumo de energia elétrica de um empreendimento situado no estado de Mato Grosso, Brasil. A partir de séries médias temporais de irradiação solar disponibilizadas pelo banco de dados do Atlas Brasileiro de Energia Solar, foi possível calcular a quantidade de energia elétrica a ser produzida no local através da implantação de painéis fotovoltaicos.

\section{METODOLOGIA}

\section{Área de estudo}

A avaliação quanto ao uso da energia solar para suprimento energético foi aplicada em uma fazenda, denominada Fazenda Tenfen, localizada no município Sorriso/MS, com cultivo de grãos - soja e milho - e que tem como atividade principal a criação de aves para corte, desenvolvida desde o ano de 2010. A fazenda possui área total de 90 hectares e, para execução de suas atividades, conta com oito galpões de $2.070 \mathrm{~m}^{2}$ cada, uma sede de escritório, um almoxarifado e quatro residências.

A utilização de eletricidade se dá principalmente para refrigeração dos aviários, mantendo a temperatura na faixa entre $30^{\circ} \mathrm{C}$ e $32^{\circ} \mathrm{C}$, dependendo da fase de vida do animal. Ainda, a energia é utilizada para distribuição de ração nos comedouros, funcionamento da bomba de captação de água do poço artesiano e uso nas residências e escritório. Há relatos do proprietário acerca de diversas quedas de energia na região, o que implica em consequências negativas para sua atividade.

Para instalação dos módulos fotovoltaicos do SFVCR, serão considerados os telhados dos barracões, 
área principal de interesse, onde será avaliada a definição quanto a disposição do sistema, considerando apenas um único galpão ou subdivisão entre os demais, visando não comprometer as estruturas e estética da construção.

\section{Coleta de dados}

Os principais fatores que envolvem o dimensionamento de um sistema fotovoltaico são: orientação dos módulos, disponibilidade de área e disponibilidade do recurso solar, e demanda a ser atendida (PINHO et al., 2014). Assim, o desenvolvimento deste estudo contará com o levantamento de informações e coleta de dados para atendimento aos itens citados, visando obter êxito e veracidade nos resultados alcançados.

\section{Consumo de Energia}

O consumo energético na unidade kWh é disponibilizado nas faturas de energia emitidas pela concessionária local. No caso do município Sorriso no Mato Grosso, a responsável pela distribuição de energia elétrica é a Energisa Mato Grosso, uma das empresas distribuidoras do Grupo Energisa. Para o desenvolvimento dos cálculos que compõe o dimensionamento do SFVCR, serão utilizados basicamente dois dados, sendo eles o Consumo em kWh - Ponta e o Consumo em kWh - Fora Ponta. Os valores descritos se subdividem de acordo com os horários da maior e menor consumo de energia elétrica definidos pela concessionaria local, sendo o de maior consumo chamado de Horário Ponta, fixado entre às $18 \mathrm{~h}$ e $21 \mathrm{~h}$, possuindo o valor do kWh mais elevado, e o Horário Fora Ponta, aquele de menor consumo na região. A seção adiante irá discutir acerca dos cenários adotados neste estudo considerando o consumo durante os dois horários.

\section{Irradiação Solar}

A partir dos dados disponibilizados pelo Atlas Brasileiro de Energia Solar, é possível identificar os valores de irradiação incidentes nas coordenadas geográficas de interesse, em $\mathrm{kWh} / \mathrm{m}^{2}$.dia. Tais dados são estimativas da irradiação nos planos global horizontal, o qual se refere à energia solar recebida por uma superfície posicionada horizontalmente no solo, e incidente sobre um plano inclinado, quando a superfície que recebe a energia solar está posicionada com inclinação igual à da latitude local (PEREIRA et al., 2017). Segundo Pereira et al. (2006), a máxima captação da energia solar por um sistema fotovoltaico se dá no último caso, quando há equivalência entre os valores de latitude e inclinação do plano de captação.

Para avaliar o desempenho de projetos solares, são considerados os valores de irradiação dos dias típicos. Segundo Siqueira et al. (2005), um dia típico é caracterizado de acordo com sua frequência de ocorrência no mês ou estação do ano em questão, o que representa as condições mais significativas ao longo do período. A condição do céu e variação dos dias e meses influenciam na irradiação solar que atinge a superfície, o que justifica a alteração da irradiação ao longo do ano no mesmo ponto de coordenada, bem como diferencia o valor obtido para os planos global horizontal e plano inclinado (PEREIRA et al., 2017; SORGATO et al., 2013). 


\section{Dimensionamento do SFVCR}

A fim de embasar estudos que permitam a real implantação do sistema fotovoltaico para geração de energia elétrica no empreendimento, foi realizado o dimensionamento do Sistema Fotovoltaico Conectado à Rede com escolha dos equipamentos e especificação dos inversores.

\section{Cenários para Aplicação do SFVCR}

Levando em consideração o consumo de energia a partir das atividades desenvolvidas na granja, bem como considerando que a geração de energia elétrica a partir do sol ocorre apenas durante o período com incidência dos raios solares, ou seja, no horário fora de ponta, e, ainda, analisando a área para instalação dos módulos fotovoltaicos e características técnicas dos componentes do sistema, serão estabelecidos cenários para o desenvolvimento deste estudo.

De maneira geral, serão estudados dois cenários principais, os quais consideram a geração de energia que visa atender apenas a demanda no horário fora de ponta, ou seja, quando há incidência dos raios solares nos módulos e consequente produção de energia elétrica, e um segundo cálculo para atender a somatória total da demanda energética do empreendimento, a energia consumida nos horários de ponta e fora de ponta.

Os cenários adotados visam a compensação da energia consumida apenas considerando o fator kWh, ou seja, a intenção é gerar toda energia que é consumida pelas atividades do empreendimento, sem considerar a tarifa diferenciada para ambos os horários. Caso este terceiro cenário fosse considerado para o estudo, com objetivo de produção de energia para zerar o custo dispendido com o abastecimento elétrico, a parcela de energia a ser gerada no horário de ponta levaria em consideração o valor mais alto da tarifa, sendo para o local de estudo definido pela Energisa em $\mathrm{R} \$ 1,29$ para o consumo em horário ponta, e $\mathrm{R} \$ 0,27$ para o consumo no horário fora de ponta. Assim, proporcionalmente o sistema deveria gerar 4,83 vezes mais energia para que a fatura de energia não contabilizasse o valor consumido no horário de ponta.

\section{Dimensionamento do SFVCR}

Para o dimensionamento do SFVCR, serão utilizadas equações matemáticas e propostas metodológicas segundo Urbanetz Junior (2010) e Alves (2008), alimentadas pelos dados coletados quanto ao consumo de energia elétrica mensal do local de estudo e valores de irradiação solar nas coordenadas de interesse. A definição que norteará a próxima etapa de escolha dos componentes do sistema e sua disposição na área disponível, é a potência necessária para atender a demanda energética das atividades do empreendimento, segundo Equação 1 a seguir (URBANETZ JUNIOR, 2010):

$$
P_{F V}=\frac{E \cdot G}{H_{T O T} \cdot P R} \quad \text { Equação } 1
$$


$G$ - Irradiância na condição STC $\left(1.000 \mathrm{~W} / \mathrm{m}^{2}\right)$

$H_{T O T}$ - Irradiação média diária em $\mathrm{kWh} / \mathrm{m}^{2}$

$P R$ - Performance ratio do SFVCR (70\% a 80\%)

A partir dos dados fornecidos pelas faturas de energia, disponibilizadas pela concessionária local, foi possível definir a demanda média de energia diária que o sistema deveria gerar. Este valor foi obtido a partir da divisão da média do consumo dos doze meses pelos 365 dias do ano. Levando em consideração os cenários que serão elaborados, será definida uma potência para atendimento da somatória da demanda nos horários de ponta e fora de ponta, e também para atendimento apenas da demanda no horário fora de ponta.

Se tratando da irradiação, o Atlas Brasileiro de Energia Solar disponibiliza os valores na unidade $\mathrm{kWh} / \mathrm{m}^{2}$.dia, foi possível estabelecer as médias de irradiação mensal e anual. Considerando que esta pesquisa se baseia no consumo dos doze meses do ano de 2017, foi definida a média diária anual de irradiação para aplicação na equação.

Segundo Rüther (2004), a irradiância na condição STC ${ }^{1}$, denominada Constante Solar ( $G_{\text {extraterrestre ou }}$ $\mathrm{G}_{\mathrm{AMO}}$ ), é a intensidade de radiação que chega à superfície da Terra às 12:00 horas, após atravessar a atmosfera e sofrer processos de absorção por gases de ozônio, gás carbônico, água e espalhamento pelo ar. No topo da atmosfera a radiação solar é reduzida a $1.353 \mathrm{~W} / \mathrm{m}^{2}$, já a intensidade de radiação solar que efetivamente chega à superfície é da ordem de $1.000 \mathrm{~W} / \mathrm{m}^{2}$ (RÜTHER, 2004).

A Performance ratio (do Ing.: 'performance' = rendimento, resultado e 'ratio' = relação, proporção) é uma das unidades internacionais de medida para avaliação do rendimento de um sistema fotovoltaico, designando a relação entre a produção de energia real e teórica. O cálculo da Performance ratio (PR) considera as perdas do sistema fotovoltaico devido à temperatura das células, utilização incompleta da irradiação e ineficiências ou falhas dos componentes do sistema. Assim, a PR pode ser utilizada como indicador comparativo entre sistemas com projetos semelhantes, mas com diferenças de layout ou instalados em locais distintos, bem como pode comparar o mesmo sistema ao longo dos anos (REICH et al., 2011). A PR é fornecida em porcentagem, onde quanto mais próximo do $100 \%$, mais eficiente é o sistema. Assim, para o desenvolvimento deste estudo, será assumido o valor de $75 \%$, valor médio entre os $70 \%$ e $80 \%$ de rendimento estabelecidos para sistemas fotovoltaicos (SMA, 2017).

Definida a potência do sistema, o próximo passo foi a escolha dos principais componentes do SFVCR, ou seja, módulos fotovoltaicos e inversores. Segundo Serrão (2010), para os sistemas conectados à rede, é necessário a inclusão de equipamentos para proteção aos painéis. Porém, considerando o escopo desta pesquisa, demais equipamentos elétricos como medidores, equipamentos de proteção e quantificação de cabeamento do sistema não serão pauta de discussão. Vale ressaltar que todos são necessários para real implantação, apenas não serão definidos neste momento.

A escolha dos equipamentos tomará como base pesquisas em sites de fornecedores credenciados na Agência Especial de Financiamento Industrial (FINAME), um programa de financiamento para aquisição de

\footnotetext{
${ }^{1}$ STC (Standard Test Conditions) - Condições de Padrão Teste, que são: irradiação solar $1000 \mathrm{~W} / \mathrm{m}^{2}$, temperatura da célula em $25^{\circ} \mathrm{C}$, distribuição espectral em AM 1,5 e incidência normal (RAMOS, 2006).
} 
máquinas e equipamentos nacionais desenvolvido pelo Banco Nacional de Desenvolvimento (BNDES). Ainda, a escolha destes equipamentos será definida principalmente com base na potência instalada do sistema, preço, área disponível para implantação e disposição no local visando não interferir na estética, bem como para preservar a estrutura física dos barracões.

Levando em conta o desenvolvimento deste estudo, foram elencadas as principais informações técnicas necessárias para o dimensionamento de um sistema fotovoltaico conectado à rede. Tais informações são disponibilizadas através das fichas de especificação técnica fornecidas pelo fabricante do equipamento em questão. O Quadro 1 compila alguns dos dados referentes ao painel e ao inversor adotados para este estudo.

Quadro 1: Especificações técnicas de painéis fotovoltaicos e inversores.

\begin{tabular}{|l|l|l|l|}
\hline Painel Fotovoltaico & Inversor & Unidade \\
\hline Dado & Unidade & Dado & V \\
\hline Potência Nominal & W & Tensão Máxima de Entrada & A \\
\hline Tensão de Operação & V & Corrente Máxima de Entrada & W \\
\hline Tensão de Circuito Aberto & V & Potência Nominal de Saída & V \\
\hline Corrente de Curto Circuito & A & Faixa de Tensão do MPP & - \\
\hline Dimensões & $\mathrm{m}$ & - & \\
\hline
\end{tabular}

Após a escolha da marca e modelo do módulo fotovoltaico a ser utilizado, a definição do número de módulos necessário para atingir a potência de geração do sistema pode ser estabelecida com o quociente entre a potência requerida e a potência de cada módulo, a qual é obtida a partir da ficha de especificação técnica do produto, conforme demonstra a Equação 2 (ALVES, 2008).

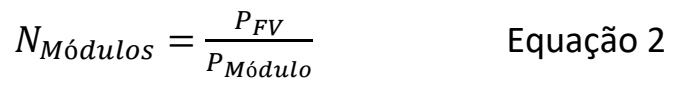

Onde:

N_Módulos - Número de módulos necessários para o SFVCR P_FV - Potência instalada necessária em Wp

P_Módulo - Potência de cada módulo fotovoltaico em W

A partir da quantidade de módulos que irá compor o sistema, foi possível calcular a área total que será necessária no local almejado para implantação, de acordo com as dimensões especificadas na ficha técnica do painel selecionado. A área total dos módulos influenciará na definição quanto à disposição dos módulos fotovoltaicos nos barracões da granja.

O tipo de ligação elétrica entre os módulos, ou seja, em série ou paralelo, visando à formação dos painéis fotovoltaicos a serem conectados nos inversores, deve ser definida observando alguns critérios. Para ligações em série, o número máximo de módulos deve considerar que a tensão em circuito aberto do gerador deve ser menor do que a tensão máxima de corrente contínua do inversor, assim, garante-se que o sistema irá operar dentro da eficiência proposta pelo equipamento, e evita possíveis danos tanto ao inversor quanto ao sistema (ALVES, 2008). Segundo Alves (2008), a Equação 3 apresenta o número máximo de módulos que podem ser ligados em série.

$$
N_{\max } \leq \frac{U_{\max I N V}}{U_{\text {Módulo }}} \quad \text { Equação } 3
$$




$$
U_{M o ́ d u l o}-\text { Tensão do módulo em V }
$$

Já com relação ao limite de ligações em paralelo, calculou-se o quociente entre os valores máximos de corrente do inversor e da corrente nominal dos módulos ligados em série, conforme exposto na Equação 4. No caso de operação do sistema em situação adversa, o inversor pode apresentar falhas precoces e até mesmo ser inutilizado (ALVES, 2008).

$$
N_{\text {max }} \leq \frac{I_{\max I N V}}{I_{\text {Módulo em série }}} \quad \text { Equação } 4
$$

$N_{\text {max }}$ - Número máximo de módulos para ligações em paralelo $I_{\text {max INV }}$ - Corrente contínua máxima do inversor em A

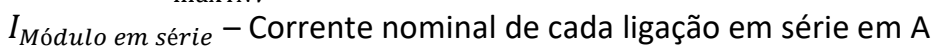

Finalmente, o SFVCR deve contar com um medidor de energia capaz de medir a geração a partir do sistema que abastecerá a rede pública, bem como deve possibilitar a medição da energia que é fornecida pela concessionária local, objetivando monitoramento mensal no que se refere à compensação energética definida pela Resolução Normativa da ANEEL. Este equipamento é de extrema importância, um componente fundamental nos SVFCR, porém, definições quanto escolha de medidor e instalação não serão abordadas no desenvolvimento deste estudo.

\section{Avaliação dos Impactos Ambientais}

Tendo em vista a complexidade envolvida em uma análise de ciclo de vida, este estudo seguiu a premissa utilizada por Mariano (2017), onde foi verificada a quantidade de dióxido de carbono que deixa de ser emitida para a atmosfera a partir da geração de energia elétrica por fonte solar. Visando reduzir a vulnerabilidade das nações à escassez de energia e diminuir as emissões de gases de efeito estufa, é necessária a inclusão de geração de energia a partir do vento, do sol e das ondas na matriz energética mundial. A minimização da dependência quanto ao uso de fontes não renováveis de energia é imprescindível para mitigar as mudanças climáticas (CARVALHO, 2014, citado por GIDDENS, 2010).

A estimativa quanto às emissões evitadas a partir da geração de energia elétrica é obtida a partir da multiplicação do fator de emissão médio de $\mathrm{CO}_{2}$ para energia elétrica - calculado com base nos dados de usinas em operação do Sistema Interligado Nacional - pela quantidade de energia consumida no local de interesse (MTCIC, 2018).

\section{RESULTADOS E DISCUSSÃO}

\section{Consumo mensal}

Segundo dados disponibilizados nas contas de energia elétrica do empreendimento, foram identificados os valores de energia consumidos mensalmente durante o ano de 2017, apresentados na Tabela 1 a seguir. Os valores descritos subdividem-se de acordo com os horários de ponta e fora de ponta, ou seja, horários de maior consumo e horários de menor consumo na região, indicados na unidade kWh/mês. 
Tabela 1: Consumo de energia elétrica em kWh/mês durante o ano de 2017.

\begin{tabular}{|c|c|c|c|}
\hline Mês & $\begin{array}{l}\text { Consumo Ponta } \\
\text { (Período compreendido entre } 18 \text { e } 21 \mathrm{hrs} \text { ) (kWh/mês) }\end{array}$ & $\begin{array}{l}\text { Consumo Fora de Ponta } \\
\text { (kWh/mês) }\end{array}$ & $\begin{array}{l}\text { Total } \\
\text { (kWh/mês) }\end{array}$ \\
\hline Janeiro & 1.561 & 17.956 & 19.517 \\
\hline Fevereiro & 1.447 & 16.103 & 17.550 \\
\hline Março & 1.592 & 19.082 & 20.674 \\
\hline Abril & 2.720 & 27.848 & 30.568 \\
\hline Maio & 516 & 5.614 & 6.130 \\
\hline Junho & 1.801 & 23.107 & 24.908 \\
\hline Julho & 584 & 5.477 & 6.061 \\
\hline Agosto & 665 & 11.266 & 11.931 \\
\hline Setembro & 2.647 & 35.231 & 37.878 \\
\hline Outubro & 374 & 4.742 & 5.116 \\
\hline Novembro & 2.610 & 31.198 & 33.808 \\
\hline Dezembro & 1.258 & 12.582 & 13.840 \\
\hline TOTAL & 17.775 & 210.206 & 227.981 \\
\hline
\end{tabular}

Fonte: Energisa (2018).

O Gráfico 1 ilustra o consumo mensal dos horários de ponta e fora de ponta, frente ao consumo total registrado pela concessionária local Energisa.

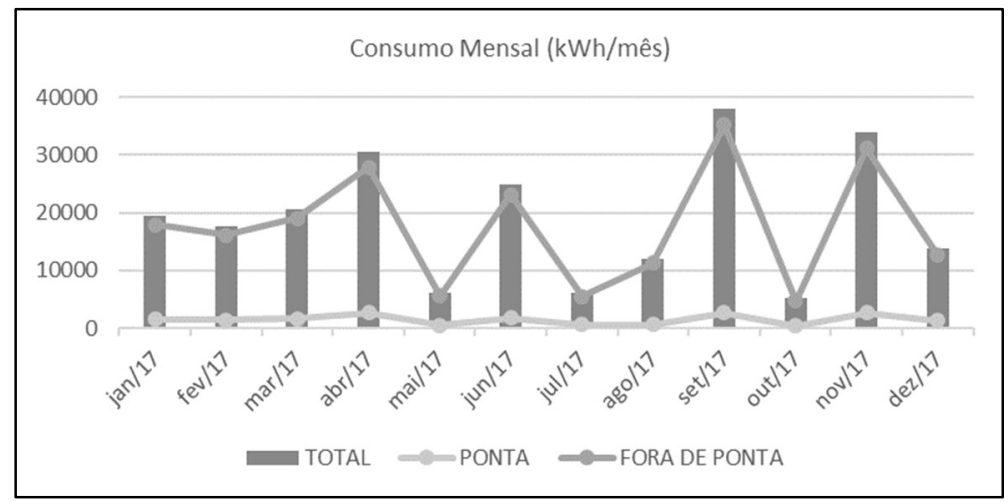

Gráfico 1: Consumo mensal total de energia elétrica.

\section{Irradiação solar}

Como pode ser observado através da Tabela 1 e Gráfico 2, os meses de maior e menor consumo durante o ano de 2017 foram, respectivamente, os meses setembro e outubro. A demanda de energia é variável, de acordo com a condição climática média do mês e atividades para produção dos lotes. Conforme exposto, a intensidade da irradiação solar que atinge a superfície varia de acordo com a inclinação do plano, ou seja, possui valores diferentes quando se considera o plano global horizontal ou plano inclinado. Considerando a proximidade entre a inclinação da cobertura dos galpões do empreendimento - em torno de 13ㅇ- com a latitude do ponto de medições do Atlas Brasileiro de Energia Solar no valor de $12,76^{\circ}$, para os cálculos será considerado o valor de irradiação em destaque do plano inclinado, descrito na Tabela 2. A Fazenda se encontra sob as coordenadas 1244'15"S e 5554'24"O.

Tabela 2: Valores de irradiação solar em $\mathrm{kWh} / \mathrm{m}^{2}$.dia.

\begin{tabular}{|l|l|l|}
\hline Mês & Plano Global $\left(\mathrm{kWh} / \mathrm{m}^{2}\right.$.dia) & Plano Inclinado $\left(\mathrm{kWh} / \mathrm{m}^{2}\right.$.dia) \\
\hline Janeiro & 5,18 & $\mathbf{4 , 8 3}$ \\
\hline Fevereiro & 4,97 & $\mathbf{4 , 8 0}$ \\
\hline Março & 4,96 & $\mathbf{4 , 9 9}$ \\
\hline Abril & 4,88 & $\mathbf{5 , 1 7}$ \\
\hline Maio & 4,67 & $\mathbf{5 , 1 7}$ \\
\hline Junho & 4,57 & $\mathbf{5 , 1 7}$ \\
\hline
\end{tabular}




\begin{tabular}{|l|l|l|}
\hline Julho & 4,76 & $\mathbf{5 , 3 2}$ \\
\hline Agosto & 5,21 & $\mathbf{5 , 6 0}$ \\
\hline Setembro & 5,12 & $\mathbf{5 , 2 3}$ \\
\hline Outubro & 5,27 & $\mathbf{5 , 1 3}$ \\
\hline Novembro & 5,15 & $\mathbf{4 , 8 4}$ \\
\hline Dezembro & 5,22 & $\mathbf{4 , 8 2}$ \\
\hline MÉDIA ANUAL & 5,00 & $\mathbf{5 , 0 9}$ \\
\hline
\end{tabular}

Fonte: Pereira et al. (2017).

A partir dos dados de irradiação solar descritos na Tabela 2, percebe-se que não há um padrão entre os valores de Plano Global e Inclinado, além disso, para o Plano Inclinado, observa-se que o maior valor de irradiação solar foi obtido durante o mês de agosto, e o menor em Fevereiro.

\section{Dimensionamento do SFVCR}

\section{Cálculo da potência}

Seguindo a metodologia adotada, foi definida a potência necessária para o sistema, a fim de que fosse gerada energia elétrica o suficiente para suprir a demanda energética das atividades do empreendimento, seguindo a premissa dos dois cenários principais. Para cálculo da potência foram utilizados os dados obtidos nas faturas de energia, segundo Tabela 1, bem como valores de irradiação descritos na Tabela 2.

Cenário 1: Consumo no horário fora ponta:

$$
P_{F V}=\frac{E . G}{H_{T O T} \cdot P R} \quad P_{F V}=\frac{\left(\frac{210.206 \mathrm{kWh}}{365 \mathrm{dias}}\right) 1 \mathrm{~kW} / \mathrm{m}^{2}}{5,09 \frac{\mathrm{kWh}}{\mathrm{m}^{2} \cdot \mathrm{dia}} \times 75 \%} \quad P_{F V}=150,860 \mathrm{kWp}
$$

Cenário 2: Consumo no horário ponta e fora ponta:

$$
P_{F V}=\frac{E . G}{H_{T O T} \cdot P R} \quad P_{F V}=\frac{\left(\frac{227.981 \mathrm{kWh}}{365 \mathrm{dias}}\right) 1 \mathrm{~kW} / \mathrm{m}^{2}}{5,09 \frac{\mathrm{kWh}}{\mathrm{m}^{2} \cdot \mathrm{dia}} \times 75 \%} \quad P_{F V}=163,616 \mathrm{kWp}
$$

\section{Equipamentos}

A definição dos equipamentos principais para composição do sistema fotovoltaico se deu a partir da avaliação quanto ao atendimento à potência instalada necessária e preços disponíveis no mercado, recaindo sobre a relação preço/potência. Os Quadros 2 e 3 apresentam os módulos fotovoltaicos e inversores que farão parte do sistema em tela, além de algumas de suas informações técnicas descritas em suas respectivas fichas de especificações.

Quadro 2: Especificações técnicas do módulo fotovoltaico.

\begin{tabular}{|l|l|}
\hline Módulo Fotovoltaico & Canadian Solar \\
\hline Marca & $\begin{array}{l}\text { KuPower } \\
\text { High Efficiency Poly }{ }^{G E N 3} \text { Module CS3K-285 }\end{array}$ \\
\hline Modelo & $285 \mathrm{~W}$ \\
\hline Potência Nominal & $31,4 \mathrm{~V}$ \\
\hline Tensão de Operação & $38,1 \mathrm{~V}$ \\
\hline Tensão de Circuito Aberto & $9,56 \mathrm{~A}$ \\
\hline Corrente de Curto Circuito & $1,675 \times 0,992 \times 0,040 \mathrm{~m}$ \\
\hline Dimensões &
\end{tabular}


Quadro 3: Especificações técnicas do inversor.

\begin{tabular}{|l|l|}
\hline Inversor & Sungrow \\
\hline Marca & $\begin{array}{l}\text { SG60KTL } \\
\text { String Inverter }\end{array}$ \\
\hline Modelo & $1000 \mathrm{~V}$ \\
\hline Tensão Máxima de Entrada & $120 \mathrm{~A}$ \\
\hline Corrente Máxima de Entrada & $60000 \mathrm{~W}$ \\
\hline Potência Nominal de Saída & $570-950 \mathrm{~V}$ \\
\hline Faixa de Tensão do MPP & \\
\hline
\end{tabular}

\section{Disposição do SFVCR}

Após definições quanto a potência do sistema necessária para geração de energia elétrica e consequente abastecimento do empreendimento, bem como escolha dos componentes do sistema fotovoltaico, esta sessão visa realizar a verificação acerca da disposição dos painéis de acordo com a área disponível. A partir da substituição dos dados na Equação 2, foi possível determinar o número de painéis e área necessária para o sistema observando os dois cenários.

Cenário 1:

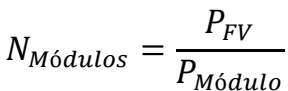

$$
\begin{aligned}
& N_{\text {Módulos }}=\frac{150.859,685 \mathrm{Wp}}{285 \mathrm{~W}} \\
& N_{\text {Módulos }} \cong 530 \text { unidades } \\
& \text { Área }=530 \times(1,675 \times 0,992) \cong 880 m^{2}
\end{aligned}
$$

Cenário 2:

$$
\begin{aligned}
& N_{\text {Módulos }}=\frac{P_{F V}}{P_{\text {Módulo }}} \\
& N_{\text {Módulos }}=\frac{163.616,366 \mathrm{Wp}}{285 \mathrm{~W}} \\
& N_{\text {Módulos }} \cong 574 \text { unidades } \\
& \text { Área }=574 x(1,675 \times 0,992) \cong 954 \mathrm{~m}^{2}
\end{aligned}
$$

Considerando a disposição dos oito barracões na área de estudo em dois conjuntos de quatro, cada um com $2.070 \mathrm{~m}^{2}$ de área, para o dimensionamento deste sistema foi adotada a área da cobertura de quatro barracões para instalação. Ainda, de acordo com as características do inversor escolhido, foram utilizados dois inversores para suprir a demanda necessária.

Tendo em vista que para ambos os cenários os equipamentos utilizados foram os mesmos, as Equações 3 e 4 visam estabelecer o limite de operação quanto a configuração das ligações elétricas entre cada módulo e dos painéis com o inversor. Cada conjunto de módulos fotovoltaicos conectados em série é denominado String Box (RÜTHER, 2004), e, para o sistema em questão, o número máximo de ligações em série foi obtido segundo a equação abaixo:

$$
N_{\text {max }} \leq \frac{U_{\text {max INV }}}{U_{\text {Módulo }}} \quad N_{\max } \leq \frac{1.000 \mathrm{~V}}{38,1 \mathrm{~V}} \quad N_{\text {Max }} \leq 26,25
$$

Ou seja, cada string poderá conter até 26 módulos fotovoltaicos com ligações em série. Já o número para as ligações em paralelo entre cada string é obtido conforme:

$$
N_{\text {max }} \leq \frac{I_{\max I N V}}{I_{\text {Módulo em série }}} \quad N_{\max } \leq \frac{120 \mathrm{~A}}{9,56 \mathrm{~A}} \quad N_{\text {Max }} \leq 12,55
$$

Assim, a partir das informações geradas, sejam elas: potência do sistema, equipamentos, número de 
módulos fotovoltaicos necessários e qual a disposição máxima suportada para operação dos equipamentos, foram definidas as disposições dos sistemas para os Cenários 1 e 2. O sistema dimensionado para o Cenário 1 considerou 530 módulos fotovoltaicos, os quais foram divididos entre os quatro galpões. Já para o Cenário 2, foram dispostos na área um total de 574 módulos fotovoltaicos.

\section{Cálculo da energia gerada}

Considerando que os sistemas terão, respectivamente, 530 e 574 módulos fotovoltaicos com uma potência nominal de $285 \mathrm{~W}$ cada, a potência instalada de cada projeto fica definida em 151,050kWp para o Cenário 1, e 163,590kWp para o Cenário 2, atendendo, portanto, as potências calculadas inicialmente para geração da energia demandada.

A fim de estimar a produção média de energia por mês a partir do sistema dimensionado, foi possível utilizar a Equação 1 isolando-se o valor de $E$, onde anteriormente foi preenchido com a demanda energética do empreendimento. $O H_{T O T}$ deve ser substituído pela irradiação de cada mês, de acordo com os valores informados na Tabela 2, já para a Performance ratio se mantém o valor de $75 \%$. Como a unidade do $H_{T O T}$ é dada em $\mathrm{kWh} / \mathrm{m}^{2}$.dia ${ }^{2}$, para definição do valor mensal de energia, é necessária a multiplicação do resultado pelo número de dias do mês em questão. $\mathrm{O}$ cálculo será demonstrado para o mês de janeiro nos dois cenários, já o resultado para os doze meses será apresentado na Tabela 3.

Cenário 1:

$$
\begin{gathered}
E=\frac{P_{F V} \cdot H_{T O T} \cdot P R}{G} \times \text { número de dias do mês } \\
E=\frac{(285 W \times 530 \text { módulos }) \times 4830 \frac{W h}{m^{2} \cdot \text { dia }} \times 75 \%}{1.000 \mathrm{~W} / \mathrm{m}^{2}} \times 31 \text { dias em janeiro } \\
E=16.962 .537,38 \frac{\mathrm{Wh}}{\mathrm{mês}}=16.962,54 \frac{\mathrm{kWh}}{\mathrm{mês}} \text { janeiro }
\end{gathered}
$$

Cenário 2:

$$
\begin{gathered}
E=\frac{P_{F V} \cdot H_{T O T} \cdot P R}{G} \times \text { número de dias do mês } \\
E=\frac{(285 W \times 574 \text { módulos }) \times 4830 \frac{\mathrm{Wh}}{\mathrm{m}^{2} \cdot \text { dia }} \times 75 \%}{1.000 \mathrm{~W} / \mathrm{m}^{2}} \times 31 \text { dias em janeiro } \\
E=18.370 .748,026 \frac{\mathrm{Wh}}{\mathrm{mês}}=18.370,75 \frac{\mathrm{kWh}}{\mathrm{mês}} \text { janeiro }
\end{gathered}
$$

Tabela 2: Estimativa de geração de energia elétrica em kWh/mês.

\begin{tabular}{|l|l|l|}
\hline \multirow{2}{*}{ Mês } & Geração (kWh/mês) & Cenário 2 \\
\cline { 2 - 3 } & Cenário 1 & 18.371 \\
\hline Janeiro & 16.963 & 16.490 \\
\hline Mevereiro & 15.226 & 18.979 \\
\hline Abril & 17.524 & 19.030 \\
\hline Maio & 17.571 & 19.664 \\
\hline Junho & 18.157 & 19.030 \\
\hline Julho & 17.571 & 20.234 \\
\hline Agosto & 18.683 & 21.299 \\
\hline Setembro & 19.667 & 19.250 \\
\hline Outubro & 17.775 & 19.512 \\
\hline Novembro & 18.016 & 17.815 \\
\hline Dezembro & 16.449 & 18.333 \\
\hline TOTAL & 16.927 & 228.007 \\
\hline
\end{tabular}

\footnotetext{
${ }^{2}$ Para aplicação na fórmula, o valor será convertido para $\mathrm{Wh} / \mathrm{m}^{2}$.dia, visando igualdade nas unidades.
} 
A fim de avaliar a geração de energia através do SFVCR frente ao consumo efetivo durante o ano de 2017, as Tabelas 4 e 5 apresentam a compilação dos dados obtidos a partir dos cálculos realizados para ambos os cenários, os quais também estão representados nos Gráficos 2 e 3.

Tabela 3: Consumo de energia e estimativa de geração - Cenário 1.

\begin{tabular}{|l|l|l|}
\hline Mês & Consumo Fora de Ponta (kWh/mês) & Geração (kWh/mês) \\
\hline Janeiro & 17.956 & 16.963 \\
\hline Fevereiro & 16.103 & 15.226 \\
\hline Março & 19.082 & 17.524 \\
\hline Abril & 27.848 & 17.571 \\
\hline Maio & 5.614 & 18.157 \\
\hline Junho & 23.107 & 17.571 \\
\hline Julho & 5.477 & 18.683 \\
\hline Agosto & 11.266 & 19.667 \\
\hline Setembro & 35.231 & 17.775 \\
\hline Outubro & 4.742 & 18.016 \\
\hline Novembro & 31.198 & 16.449 \\
\hline Dezembro & 12.582 & 16.927 \\
\hline TOTAL & 210.206 & 210.529 \\
\hline
\end{tabular}

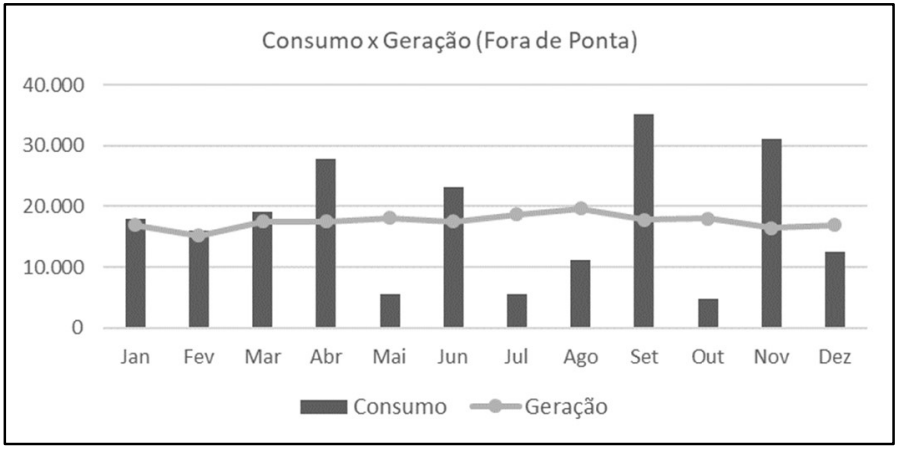

Gráfico 2: Consumo e estimativa de geração de energia para o Cenário 1.

Tabela 5: Consumo de energia e estimativa de geração - Cenário 2.

\begin{tabular}{|l|l|l|}
\hline Mês & Consumo Ponta + Fora de Ponta (kWh/mês) & Geração (kWh/mês) \\
\hline Janeiro & 19.517 & 18.371 \\
\hline Fevereiro & 17.550 & 16.490 \\
\hline Março & 20.674 & 18.979 \\
\hline Abril & 30.568 & 19.030 \\
\hline Maio & 6.130 & 19.664 \\
\hline Junho & 24.908 & 19.030 \\
\hline Julho & 6.061 & 20.234 \\
\hline Agosto & 11.931 & 21.299 \\
\hline Setembro & 37.878 & 19.250 \\
\hline Outubro & 5.116 & 19.512 \\
\hline Novembro & 33.808 & 17.815 \\
\hline Dezembro & 13.840 & 18.333 \\
\hline TOTAL & 227.981 & 228.007 \\
\hline
\end{tabular}

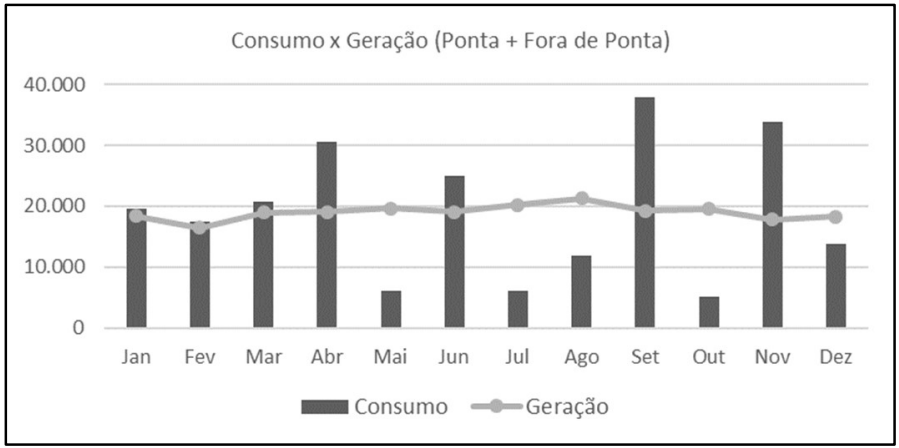

Gráfico 3: Consumo e estimativa de geração de energia para o Cenário 2. 
É possível verificar que a geração de energia a partir do sistema fotovoltaico para ambos os cenários se mantem na faixa de aproximadamente $15.000 \mathrm{kWh} / \mathrm{mês}$ a $20.000 \mathrm{kWh} / \mathrm{mês}$, já o consumo tem grandes variações ao longo dos meses. Ou seja, vezes o sistema está abastecendo a rede interligada com valores de energia que ele não irá consumir, outrora está consumindo mais energia do que produziu no mesmo período.

As atividades na granja são marcadas por períodos de limpeza onde se consome menos energia, tendo em vista que os equipamentos de alimentação e equilíbrio da temperatura não são utilizados. Ainda, os mesmos têm maior consumo quando há uma necessidade de resfriamento nos dias mais quentes, como nos meses de setembro e novembro.

\section{Estimativa de emissões evitadas}

A quarta edição do Relatório de Estimativas Anuais de Emissões de Gases de Efeito Estufa no Brasil publicado pelo MCTIC estendeu o período analisado até o ano de 2015, a partir dos dados do Terceiro Inventário que contempla a série histórica de emissões desde o ano de 1990 a 2010. O infográfico disponibilizado pelo MCTIC indica que durante o ano de 2015 foram emitidos 1.368 milhões de toneladas de $\mathrm{CO}_{2}$ eq no Brasil, destes, 33\% de responsabilidade do setor energético, ou seja, 451.440 .000 tCO 2 eq. A Tabela 6 apresenta os fatores médios de emissão de $\mathrm{CO}_{2}$ para energia elétrica, calculados a partir dos dados disponibilizados pelo SIN, num período de dez anos. O fator médio de emissões entre os anos de 2008 e 2017 foi de $0,0749 \mathrm{tCO}_{2}$ eq no setor elétrico.

Tabela 6: Fator Médio Anual de Emissões de $\mathrm{CO}_{2}$ do $\mathrm{SIN}$ em $\mathrm{tCO}_{2} / \mathrm{MWh}$.

\begin{tabular}{|l|l|l|l|l|l|l|l|l|l|l|l|}
\hline Ano & 2008 & 2009 & 2010 & 2011 & 2012 & 2013 & 2014 & 2015 & 2016 & 2017 & Média \\
\hline Fator Médio & 0,0477 & 0,0194 & 0,0532 & 0,0349 & 0,1168 & 0,0841 & 0,1368 & 0,1075 & 0,0714 & 0,0892 & 0,0749 \\
\hline
\end{tabular}

No desenvolvimento deste estudo, o dimensionamento de um sistema fotovoltaico para implantação em uma granja de aves resultou em dois projetos com potências instaladas de 151,050kWp e 163,590kWp, os quais são capazes de gerar $210.529 \mathrm{kWh} /$ ano e $228.007 \mathrm{kWh} /$ ano de energia, respectivamente. Utilizando o fator médio de emissões referente aos dez anos da Tabela 7, igual a 0,0749 $\mathrm{tCO}_{2}$ eq de emissões para cada MWh de energia gerada, a implantação do SFVCR evitaria a emissão de aproximadamente 15,8 tCO ${ }_{2}$ eq por ano para o Cenário 1, e 17,1 tCO ${ }_{2}$ eq por ano para o Cenário 2.

\section{CONCLUSÕES}

Por meio da metodologia adotada neste trabalho, foram levantados dados acerca do consumo de energia elétrica de uma granja de aves no município Sorriso/MT, utilizada para o desenvolvimento das atividades, além de dados disponibilizados pelo Atlas Brasileiro de Energia Solar acerca da irradiação solar no ponto de interesse, visando o dimensionamento de um Sistema Fotovoltaico Conectado à Rede. Ainda, de acordo com a literatura, foram levantados quais os principais aspectos socioambientais intrínsecos à tecnologia.

Foram definidos dois cenários para estudo, os quais consideram a geração de energia elétrica para suprir o consumo de energia no horário fora de ponta, e também o consumo total a partir da somatória da 
energia consumida nos horários de ponta e fora de ponta. De acordo com os cálculos realizados para dimensionar o sistema, foram definidas as potências necessárias para ambos os cenários, sendo elas, respectivamente 151,050kWp e 163,590kWp. A partir de então, os SFVCR foram configurados e os principais equipamentos definidos, sendo possível concluir que o local possui área e incidência solar suficientes para geração de energia elétrica e atendimento às necessidades energéticas do empreendimento a partir de uma fonte renovável de energia.

Acerca da avaliação quanto aos impactos ambientais, verificou-se que os impactos mais significativos se concentram durante a fase de implantação do sistema, abrangendo a fabricação dos equipamentos a serem utilizados, etapa onde são consumidos recursos naturais e há emissão de gases poluentes para a atmosfera. Segundo dados disponibilizados pelo MCTIC, a emissão de $\mathrm{CO}_{2}$ eq durante o ano de 2015 contabilizada pelo SIN foi estimada em 451.440 .000 tCO 2 eq, sendo $17,1 \mathrm{tCO}_{2}$ eq de responsabilidade do empreendimento foco deste estudo. Caso seja implantado o sistema fotovoltaico visando a compensação de energia, a contribuição da granja para minimizar as emissões do SIN seria de aproximadamente $4 \%$.

Isto posto, permite-se sugerir com uma ótica positiva a instalação da usina fotovoltaica na granja de aves. A incorporação da matriz energética com fontes alternativas de geração de energia colabora para evolução em busca do desenvolvimento sustentável, colaborando para mitigar os efeitos das mudanças climáticas no âmbito global a partir da diminuição de emissões de GEEs.

\section{REFERÊNCIAS}

ALVES, V, A. M.. Dimensionamento de Sistemas de Produção de Electricidade Baseados em Energia Solar Fotovoltaica. Dissertação (Mestrado Integrado em Engenharia Electrotécnica e de Computadores) - Faculdade de Engenharia da Universidade do Porto, Portugal, 2008.

ANEEL. Agência Nacional de Energia Elétrica. Resolução Normativa n. 482, de 17 de abril de 2012. Estabelece as condições gerais para o acesso de microgeração e minigeração distribuída aos sistemas de distribuição de energia elétrica, o sistema de compensação de energia elétrica, e dá outras providências. Brasília: ANEEL, 2012.

CARVALHO, F. I. A.. Uma Avaliação de Viabilidade Financeira no Uso da Energia Solar Fotovoltaica Apoiada pela Resolução ANEEL 482/2012 sobre Geração Distribuída no Setor Elétrico do Brasil. Dissertação (Mestrado em Administração e Controladoria) - Universidade Federal do Ceará, Fortaleza, 2014.

MARIANO, J.. Análise do Potencial da Geração de Energia Fotovoltaica para Redução dos Picos de Demanda e Contribuição Energética nas Edificações da UTFPR em Curitiba. Dissertação (Mestrado em Engenharia Civil) Universidade Tecnológica Federal do Paraná, Curitiba, 2017.

MARTINS, F. R.; GUARNIERI, R. A.; PEREIRA, E. B.. O aproveitamento da energia eólica. Revista Brasileira de Ensino de Física, v.30, n.1, p.1304, 2008. DOI: http://dx.doi.org/10.1590/S1806-11172008000100005

PEREIRA, E. B; MARTINS, F. R.; ABREU, S. L.; RÜTHER, R.. Atlas Brasileiro de Energia Solar. São José dos Campos,
2006.

PEREIRA, E. B.; MARTINS, F. R.; GONÇALVES, A. R.; COSTA, R. S.; LIMA, F. J. L.; RÜTHER, R.; ABREU, S. L.; TIEPOLO, G. M.; PEREIRA, S. V.; SOUZA, J. G.. Atlas Brasileiro de Energia Solar. 2 ed. São José dos Campos, 2017.

PINHO, J. T. GALDINO, M. A.. Manual de Engenharia para Sistemas Fotovoltaicos. Rio de Janeiro, 2014.

REICH, N. H.; MULLER, B.; ARMBRUSTER, A.; VAN SARK, W. G.; KIEFER, K.; REISE, C.. Performance Raio Revised: is $P R>90 \%$ Realistic?. Progress in Photovoltaics, n.20, p.717726, 2011.

RÜTHER, R.. Edifícios Solares Fotovoltaicos: o potencial da geração solar fotovoltaica integrada a edificações urbanas e interligada à rede elétrica pública no Brasil. Florianópolis: LABSOLAR, 2004.

SERRÃO, M. A. S.. Dimensionamento de um Sistema Fotovoltaico para uma Casa de Veraneio em Pouso da Cajaíba-Paraty. Tese (Engenharia Elétrica) - Universidade Federal do Rio de Janeiro, Rio de Janeiro, 2010.

SMA. Solar Technology AG. Performance Ratio. Technical Information, 2017.

SIQUEIRA, T. C. P. A.; AKUSTSU, M.; LOPES, J. I. E.; SOUZA, H. A.. Dados Climáticos para Avaliação de Desempenho Térmico de Edificações. REM, Ouro Preto, n.58, p.133-138, 2005. DOI: http://dx.doi.org/10.1590/S037044672005000200007 
SORGATO, M. J.; MELO, A. P., LAMBERTS, R.. Análise do Método de Simulação de Desempenho Térmico da Norma 15.575. In: ENCONTRO NACIONAL, 12; LATINOAMERICANO DE CONFORTO NO AMBIENTE CONSTRUÍDO, 8. Anais. Brasília: ENCA, 2013.

TIEPOLO, G. M.. Estudo do potencial de geração de energia elétrica através de sistemas fotovoltaicos conectados à rede no estado do Paraná. Tese (Doutorado em Engenharia de Produção e Sistemas) - Pontifícia Universidade Católica do Paraná, Curitiba, 2015.

URBANETZ JUNIOR, J.. Sistemas fotovoltaicos conectados a redes de distribuição urbanas: sua influência na qualidade da energia elétrica e análise dos parâmetros que possam afetar a conectividade. Tese (Doutorado em Engenharia Civil) - Universidade Federal de Santa Catarina, Florianópolis, 2010.

A CBPC - Companhia Brasileira de Produção Científica (CNPJ: 11.221.422/0001-03) detém os direitos materiais desta publicação. Os direitos referem-se à publicação do trabalho em qualquer parte do mundo, incluindo os direitos às renovaç̃ões, expansões e disseminações da contribuiç̃o, bem como outros direitos subsidiários. Todos os trabalhos publicados eletronicamente poderão posteriormente ser publicados em coletâneas impressas sob coordenação da Sustenere Publishing, da Companhia Brasileira de Produção Científica e seus parceiros autorizados. Os (as) autores (as) preservam os direitos autorais, mas não têm permissão para a publicação da contribuição em outro meio, impresso ou digital, em português ou em tradução. 